\title{
Editorial: Radiation-induced and oxidative DNA damages
}

\author{
Antonio Monari ${ }^{1 *}$, Elise Dumont ${ }^{2 *}$ and Chryssostomos Chatgilialoglu ${ }^{3 *}$ \\ 1 Theory-Simulatio-Modeling Group, Centre National de la Recherche Scientifique, Université de Lorraine, Nancy, France, \\ ${ }^{2}$ École normale supérieure de Lyon and Centre National de la Recherche Scientifique, Lyon, France, ${ }^{3}$ National Center for \\ Scientific Research Demokritos, Athens, Greece
}

Keywords: DNA lesions, radiation and UV induced, simulation and modeling, spectroscopies, analytical chemistry

\section{OPEN ACCESS}

Edited and reviewed by: John D. Wade,

Florey Institute of Neuroscience and

Mental Health, Australia

*Correspondence: Antonio Monari, antonio.monari@cbt.uhp-nancy.fr; Elise Dumont elise.dumont@ens-lyon.fr Chryssostomos Chatgilialoglu, c.chatgilialog/u@inn.demokritos.gr

Specialty section: This article was submitted to Chemical Biology, a section of the journal Frontiers in Chemistry

Received: 29 July 2015 Accepted: 10 August 2015 Published: 25 August 2015

Citation:

Monari A, Dumont E and Chatgilialoglu C (2015) Editorial: Radiation-induced and oxidative DNA damages. Front. Chem. 3:54. doi: 10.3389/fchem.2015.00054
The study of DNA lesions and changes, produced by interaction with UV or ionizing radiation and by the effects of oxidative stress, is a multidisciplinary research field in life sciences that has yelded exciting discoveries in the last few decades. The importance of this study is clearly enhanced by its strong societal impact, because of the connections of DNA lesions with carcinogenetic processes, which lead to the disruption or mismatching of the genetic information. As well, the controlled and selective production of lesions is a promising tool for the development of novel antitumoral strategies and drugs. The multidisciplinary character requires a combined effort from simulation and modeling studies, together with high-level experimental and analytical chemistry, spectroscopy, up to biochemical or cellular biology tools. In this Research Topic, we present a collection of articles focusing on DNA bases photoinduced and radiation damages, on the DNA interactions, on potential drug development and on the effect of radicals on DNA constituents.

The fundamental reactions of DNA constituents are taken into account for instance by the investigation of the potential mutagenic effects of $\mathrm{NO}_{2}$ radical to guanine and cytosine base pairs (Cerón-Carrasco et al., 2015) by high level simulation protocols. An ameliorated protocol has also been developed and validated to achieve a better accuracy in the determination of radiationinduced lesions in purine moieties (Terzidis et al., 2015) embedded in double- and single-strand DNA. The improved quantification of purine $5^{\prime}, 8$-cyclonucleosides resulting from the cyclization of C5 $^{\prime}$ radical on DNA has also been tackled (Terzidis and Chatgilialoglu, 2015). The association constant of lipophilic derivatives of the $5^{\prime}, 8$-cyclo- $2^{\prime}$-deoxyguanosine with $2^{\prime}$-deoxycytidine has been studied by voltammetry NMR techniques (Capobianco et al., 2015).

DNA sensitization by endogenous molecules has also been covered. The oxidative profile of copper phenanthrene chemical nucleases interacting with DNA has been presented (Molphy et al., 2015) together with a variety of biophysical data. Furthermore, Förster Resonant-EnergyTransfer has been used to prove the flexibility and dynamical behavior of short double-strand DNA sequences intercalated by organometallic compounds (Jia et al., 2015). Connected to spectroscopy a very detailed analysis of the excited state potential energy landscape of solvated guanine monophosphate has been reported with high level ab initio techniques, together with an analysis of principal photochemical pathways (Altavilla et al., 2015).

Furthermore, two reviews, one on simulation and modeling of DNA lesions (Dumont and Monari, 2015) and the other on stress-induced DNA biomarkers (Nikitaki et al., 2015), help to clearly define the state of the art of this very active scientific field.

This scientific topic is also closely related to the COST in Chemistry Action CM1201 "Biomimetic Radical Chemistry" and in particular to its Working Group 2 devoted to "Models of DNA Damages and Consequences." The COST Action members have actively participated with most of the contributions derived from the scientific collaborations ongoing in its framework. As such the Research Topic also represents a very nice overview of the effectiveness and added value brought about by the creation of a large multinational 
scientific network. Furthermore, the financial support of the COST Action CM1201 has allowed the open source publication of exciting scientific contributions from its members.

We believe that the "Radiation Induced and Oxidative DNA Damage" Research Topic nicely illustrates most of the complex and fascinating challenges that scientists working on DNA damages should tackle. At the same time, it highlights the

\section{References}

Altavilla, S. F., Segarra-Martí, J., Nenov, A., Conti, I., Rivalta, I., and Garavelli, M. (2015). Deciphering the photochemical mechanisms describing the UVinduced processes occurring in solvated guanine monophosphate. Front. Chem. 3:29. doi: 10.3389/fchem.2015.00029

Capobianco, A., Caruso, T., Fusco, S., Terzidis, M. A., Masi, A., Chatgilialoglu, C., et al. (2015). The association constant of $5^{\prime}, 8$-cyclo- $2^{\prime}$-deoxyguanosine with cytidine. Front. Chem. 3:22. doi: 10.3389/fchem.2015.00022

Cerón-Carrasco, J. P., Requena, A., Zúñiga, J., and Jacquemin, D. (2015). Mutagenic effects induced by the attack of $\mathrm{NO}_{2}$ radical to the guanine-cytosine base pair. Front. Chem. 3:13. doi: 10.3389/fchem.2015.00013

Dumont, E., and Monari, A. (2015). Understanding DNA under oxidative stress and sensitization: the role of molecular modeling. Front. Chem. 3:43. doi: 10.3389/fchem.2015.00043

Jia, F., Despax, S., Münch, J.-P., and Hébraud, P. (2015). Flexibility of short ds-DNA intercalated by a dipyridophenazine ligand. Front. Chem. 3:25. doi: 10.3389/fchem.2015.00025

Molphy, Z., Slator, C., Chatgilialoglu, C., and Kellett, A. (2015). DNA oxidation profiles of copper phenanthrene chemical nucleases. Front. Chem. 3:28. doi: $10.3389 /$ fchem. 2015.00028 scientific maturity of this field, foreseeing even more exciting perspectives that will undoubtedly blossom in the forthcoming years.

\section{Acknowledgments}

Support from COST in Chemistry CM1201 Biomimetic Radical Chemistry is gratefully acknowledged.

Nikitaki, Z., Hellweg, C. E., Georkakilas, A. G., and Ravanat, J.-L. (2015). Stress induced DNA damage biomarkers: applications and limitations. Front. Chem. 3:35. doi: 10.3389/fchem.2015.00035

Terzidis, M. A., Chatgilialoglu, C. (2015). An ameliorative protocol for the quantification of purine $5^{\prime}, 8$-cyclo-2'-deoxynucleosides in oxidized DNA. Front. Chem. 3:47. doi: 10.3389/fchem.2015.00047

Terzidis, M. A., Ferreri, C., and Chatgilialoglu, C. (2015). Radiationinduced formation of purine lesions in single and double stranded DNA: revised quantification. Front. Chem. 3:18. doi: 10.3389/fchem.2015. 00018

Conflict of Interest Statement: The authors declare that the research was conducted in the absence of any commercial or financial relationships that could be construed as a potential conflict of interest.

Copyright (C) 2015 Monari, Dumont and Chatgilialoglu. This is an open-access article distributed under the terms of the Creative Commons Attribution License (CC BY). The use, distribution or reproduction in other forums is permitted, provided the original author(s) or licensor are credited and that the original publication in this journal is cited, in accordance with accepted academic practice. No use, distribution or reproduction is permitted which does not comply with these terms. 